\title{
Simulation of Fuzzy Logic Control for DC Servo Motor using Arduino based on Matlab/Simulink
}

\author{
Munadi \\ Department of Mechanical Engineering \\ Diponegoro University \\ Tembalang, Semarang 50275, Indonesia \\ munadi@undip.ac.id
}

\author{
M. Amirullah Akbar \\ Department of Mechanical Engineering \\ Diponegoro University \\ Tembalang, Semarang 50275, Indonesia \\ amir240891@gmail.com
}

\begin{abstract}
The most widely used control strategy in industry is proportional integral derivative (PID) controller. The popularity of PID controllers can be attributed partly to their robust performance in a wide range of operating conditions and partly to their functional simplicity. One of the application is to control arm robot manipulator model by using DC servo motor as actuator. This paper presents design of PID controller of DC servo motor using automated PID tuning by sisotool for higher order system and implement to the Arduino Mega 2560 via potentiometer by using Simulink Support Package for Arduino Hardware in MATLAB/Simulink. A better design of controller using fuzzy logic controller (FLC) is proposed. Simulation results are demonstrated. Performance analysis shows the effectiveness of the proposed Fuzzy logic controller as compared to the PID controller.
\end{abstract}

Keywords- PID Controler, Fuzzy Logic Controler, Sisotool, Fuzzy Logic Toolbox, DC Servo Motor

\section{INTRODUCTION}

Industrial systems with high-efficiency and great performance have taken more advantages of robot technology. The large number of control research and many control applications were presented during the last years, concentrated on control of robotic systems. Robot manipulator field is one of the interested fields in industrial, educational and medical applications. It works in unpredictable, hazard and inhospitable circumstances which human cannot reach, such as, working in nuclear or chemical reactors is very dangerous, while when a robot instead human it involves no risk to human life [1]. Therefore, modeling and analysis system responses of DC servo motor as actuators in robot manipulators and applying control techniques such as FLC are very important before using them in these circumstances to a smooth precision movement of arm robot manipulator model. In some literature said that FLC have better stability and small overshoot [2].

This paper has two main contributions. Firstly, a PID controller has been designed for higher order system using automated PID tuning by using Matlab's SISO design tool. Secondly, for the same system a fuzzy logic controller has been proposed by using Fuzzy Logic Toolbox to design the Fuzzy Inference System (FIS). The simulation results for a higher order systems will be demonstrated both for PID and fuzzy logic control. A performance comparison such as response system between automated tuned PID controller and the proposed fuzzy logic controller is presented. The paper will be organized as follows, Section-II describes the arm robot manipulator model. Section-III describes the design and value of transfer function of DC servo motor. Section-IV describes the design of PID controller for DC servo motor. Section V presents design the FIS of FLC for DC servo motor using Fuzzy Logic Toolbox in MATLAB/Simulink. Section VI finally conclusion closes the paper.

\section{ARM ROBOT MANIPULATOR MODEL}

In order to show the effectiveness of the fuzzy logic controller to acquire an accurate tracking, we will verify the controller by simulation on the arm robot manipulator model that is shown in Fig. 1. This model represents the scale arm robot manipulator that consists of five degree of freedom and it has been designed as one of an experimental platform for research and education in our laboratory.

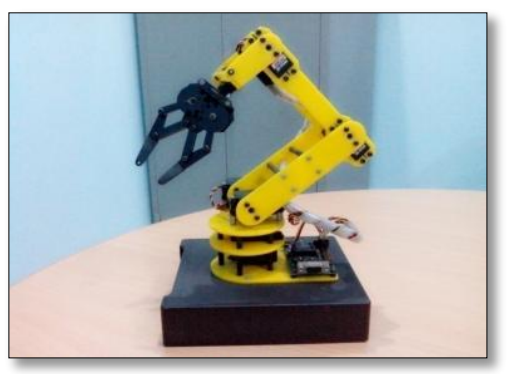

Fig. 1. Arm robot manipulator model.

Furthermore, the main component of arm robot manipulator model uses Arduino Mega 2560 in which the schematic of electronic hardware is shown in Fig. 2.

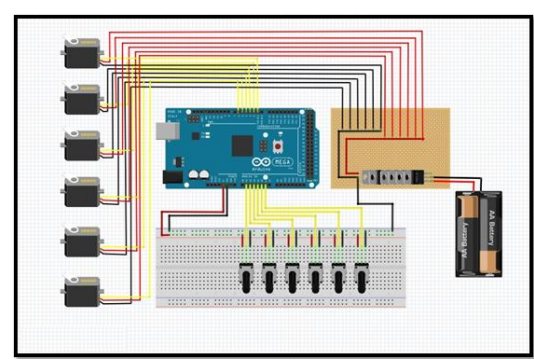

Fig. 2. Schematic of electronic hardware 


\section{DC SERVO MOTOR DESCRIPTION}

DC servo motors are most suitable for wide range speed control and are there for many adjustable speed drives. For this simulation, Fig. 3 represented the DC servo motor model. Parameters and values chosen for motor simulation can be shown at Table 1 [1].

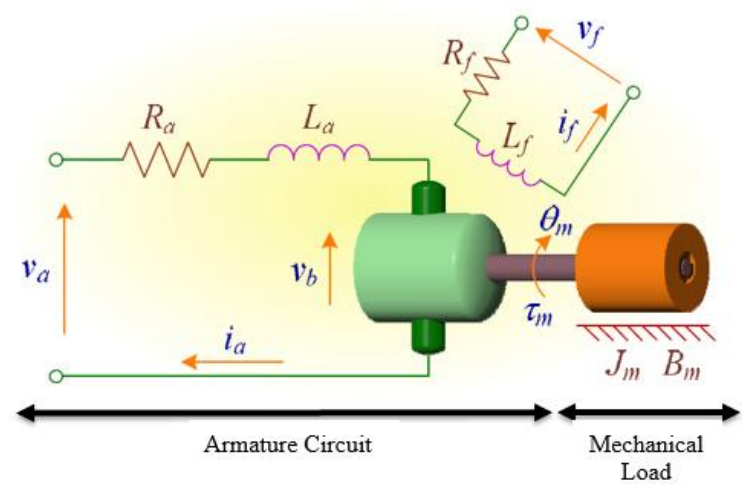

Fig. 3. Schematic representation of the DC servo motor [3].

TABLE I. DC servo motor parameter and value.

\begin{tabular}{|l|c|c|}
\hline Parameter & Nomenclature & Value \\
\hline Momen of inertia & $J_{m}$ & $0,000052 \mathrm{Kg} \cdot \mathrm{m}^{2}$ \\
\hline Friction coefficient & $B_{m}$ & $0,01 \mathrm{~N} \cdot \mathrm{ms}$ \\
\hline Back EMF constant & $K_{b}$ & $0,235 \mathrm{~V} / \mathrm{rad} \mathrm{s} \mathrm{s}^{-1}$ \\
\hline Torque constant & $K_{a}$ & $0,235 \mathrm{Nm} / \mathrm{A}$ \\
\hline Electric resistance & $R_{a}$ & $2 \mathrm{ohm}$ \\
\hline Electric inductance & $L_{a}$ & $0.23 \mathrm{H}$ \\
\hline Input voltage & $v_{a}$ & \\
\hline Back EMF voltage & $v_{b}$ & \\
\hline Armature Current & $i_{a}$ & \\
\hline Developed Torque & $\tau_{m}$ & \\
\hline Angle of motor shaft & $\theta$ & \\
\hline Load Torque & $T_{L}$ & \\
\hline
\end{tabular}

The purpose of modelling DC servo motor is to approach the actual DC servo motor [4]. Including the parameters, we can get the transfer function of DC servo motor for controlling position [1].

$$
G_{\text {position }}(s)=\frac{\theta(s)}{V_{a}(s)}=\frac{19640}{s^{3}+201 s^{2}+6290 s}
$$

Block diagram of DC servo motor is shown in Fig. 4 with zero value of load torque $\left(\mathrm{T}_{\mathrm{L}}\right)$ [5].

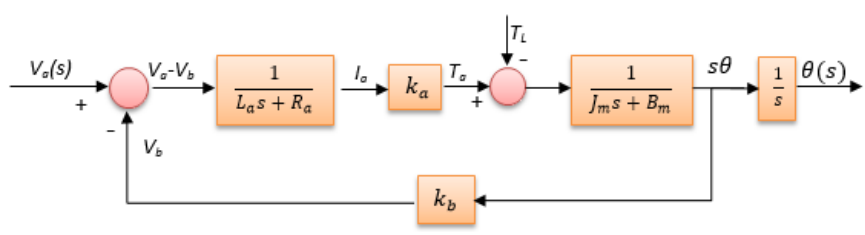

Fig. 4. Block diagram of DC servo motor.

\section{DESIGN OF THE PID CONTROLLER}

A PID controller is being designed for a higher order system with transfer function in equation (1). Before starting the simulation and implementation PID controller, we construct the design of PID controller by using Matlab's SISO design tool and analyze the stability respond systems by using root locus method [4-6]. The design architecture of Matlab's SISO design tool for automated PID tuning is shown in Fig. 5.

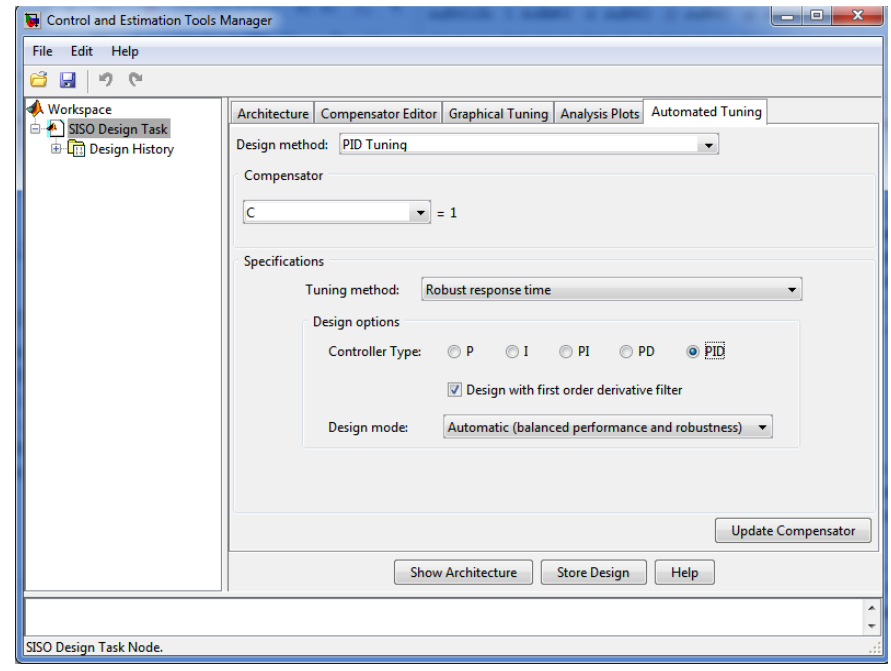

Fig.5. Automated PID tuning using Matlab's SISO design tool.

Using automated PID tuning, we can get the root locus stability for the open loop system G(s) is shown in Fig. 6.

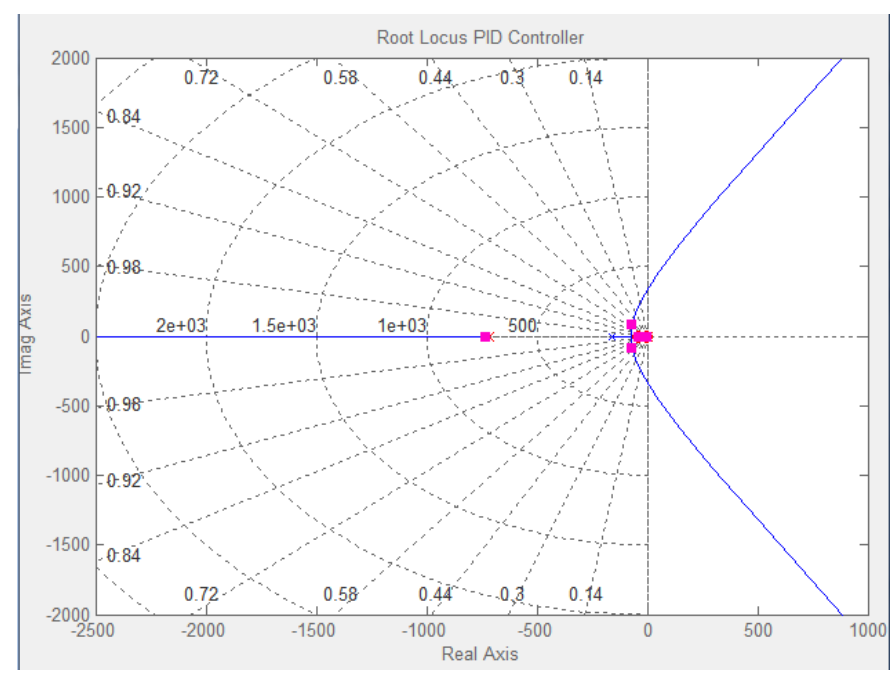

Fig. 6. Root locus of the PID controller.

There are 5 pole at Fig. 6 that represent stability for the system. The most location of the poles are located very close to the imaginary axis so that the system has a decrease value of rise time. There are two poles that not locating on real axis and imaginary axis. They indicate that there is an overshoot for the system. If the poles pass through the imaginary axis then the 
system becomes unstable. Overall based on the graph, the system is still in stable condition [5], [6].

The unit step is given for PID controller design. Fig. 7 shows the response system of its.

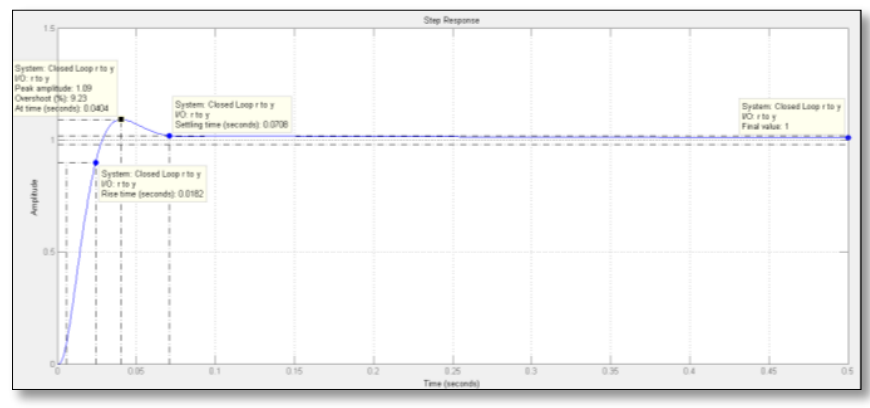

Fig. 7. Unit step response of the PID controller.

Based on Fig. 7, we can get transient parameters for the system. They are a rise time, percent overshoot, settling time, and steady state error. For the system, we can get 0.0182 second for the rise time (Tr), $9.28 \%$ for overshoot $(\mathrm{Mp}), 0.0708$ second for the settling time (Ts), and $4 \%$ steady state error (Ess).

After design and analyze the PID controller response, Fig. 8 shows the Simulink model for simulation. Given $100^{\circ}$ for the final value and 0.1 second for the step time.

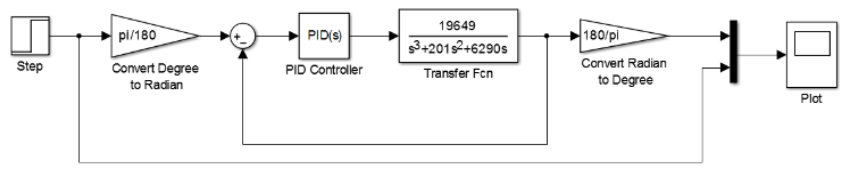

Fig. 8. Simulink model for simulating the PID controller.

Based on the Simulink model for simulating the PID controller, Fig. 9 shows the result of simulation.

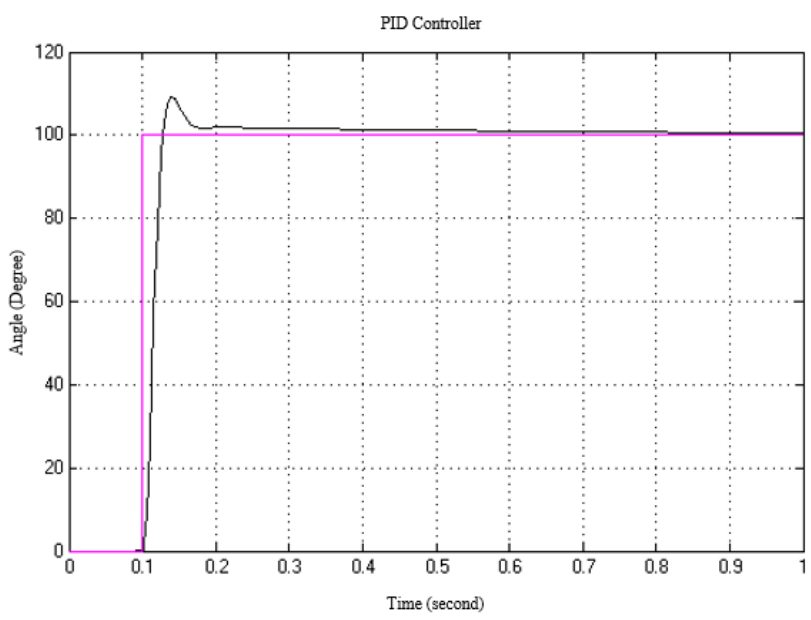

Fig. 9. Simulation result of the PID controller.
Fig. 10 shows Simulink model implementation of the PID controller to the Arduino Mega 2560 via potentiometer by using Simulink Support Package for Arduino Hardware.

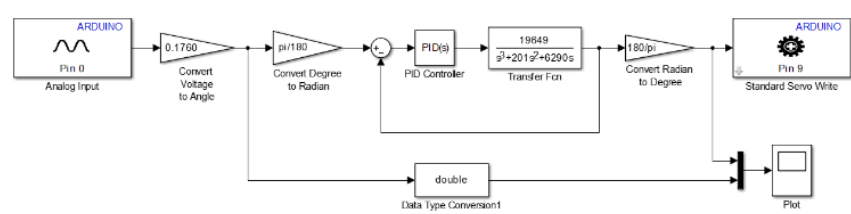

Fig. 10. Simulink model for implementing the PID controller.

Based on the Simulink model for implementing PID controller, Fig. 11 shows the result of implementation.

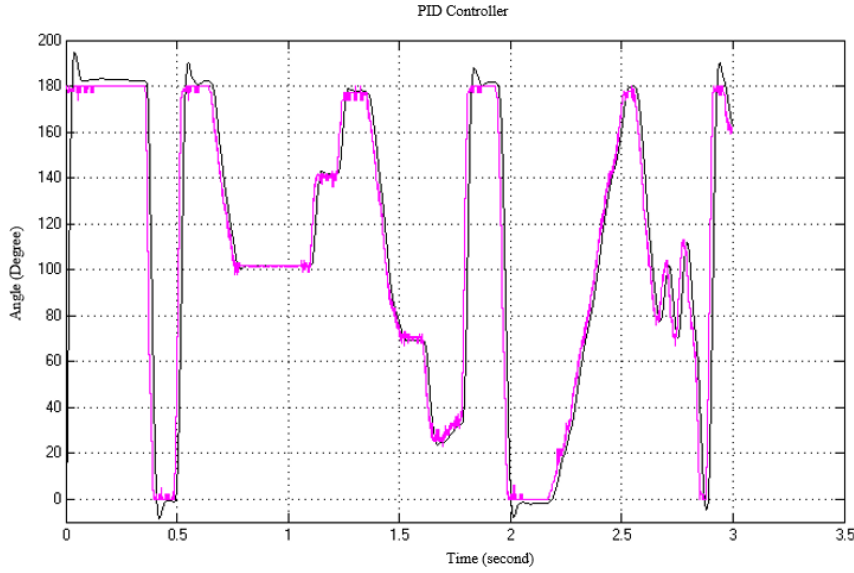

Fig. 11. Implementation result of the PID controller.

\section{DESIGN OF PROPOSED FUZZY LOGIC CONTROLLER}

A Fuzzy Logic Controller (FLC) is being designed for a higher order system with transfer function in equation (1). Before starting the simulation, we construct the design of FIS (Fuzzy Inference System) firstly by using fuzzy logic toolbox in Fig. 12 and analyze the stability respond systems by manual reading based on the graph.

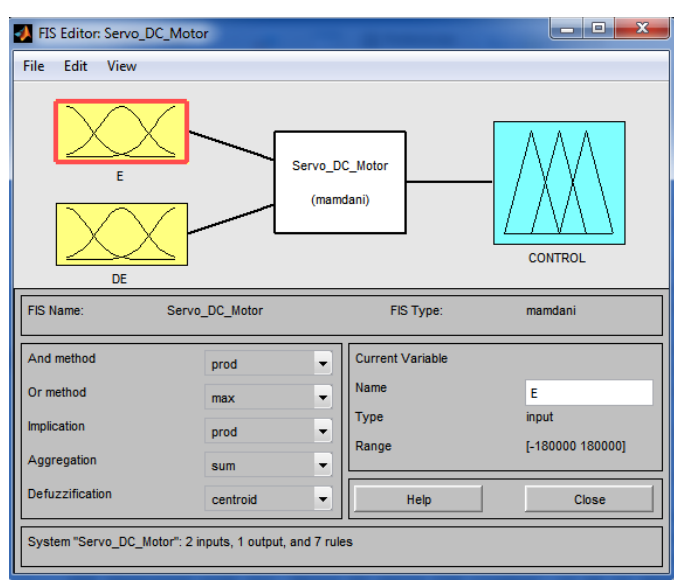

Fig. 12. The FIS editor. 
The basic FLC structure, shown in Fig. 13 contains four step. These are the fuzzification, knowledge base, decisionmaking and defuzzification units [7], [8].

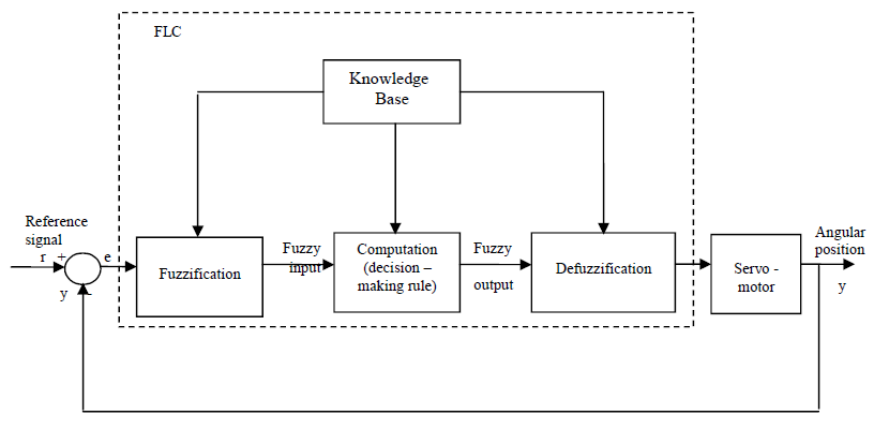

Fig.13. Basic structure of the FLC [8].

In this simulation, it is aimed to control motor position. The knowledge base contains a set of rules which construct the decision-making logic rule [8]. There are 7 rules that used at the controllers which are based on human experience and information are:

1. If $\mathrm{E}$ is $\mathrm{PL}$ then $\mathrm{CONTROL}$ is $\mathrm{PL}$

2. If $\mathrm{E}$ is $\mathrm{NL}$ then $\mathrm{CONTROL}$ is $\mathrm{NL}$

3. If $\mathrm{E}$ is $\mathrm{Z}$ and $\mathrm{DE}$ is $\mathrm{N}$ then CONTROL is $\mathrm{NM}$

4. If $E$ is $Z$ and $D E$ is $P$ then CONTROL is PM

5. If $E$ is $Z$ then $C O N T R O L$ is $Z$

6. If $E$ is NM then CONTROL is NM

7. If $\mathrm{E}$ is $\mathrm{PM}$ then CONTROL is PM

After designing the rule, we can get the surface viewer in Fig.14 that represent the rule of FLC.

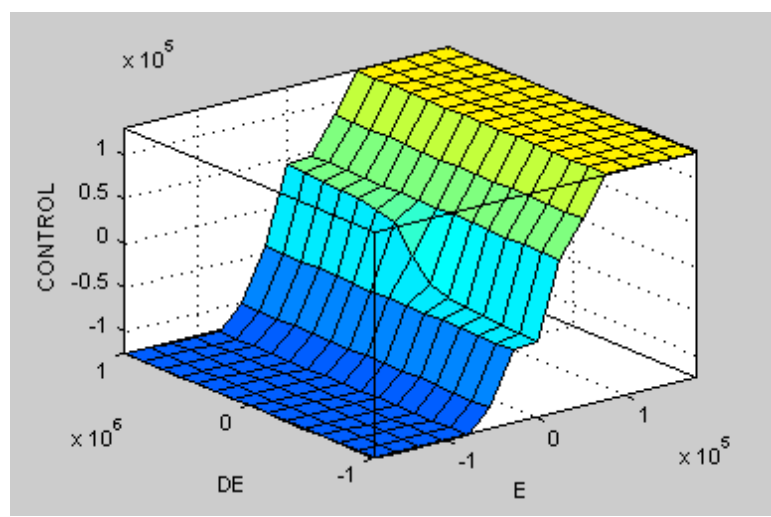

Fig. 14. 3D FLC surface.

There are two inputs and one output in FIS. Two input contains error (E) and delta error (DE) [9]. One output is a control signal to the plant. The fuzzy membership functions for the two input parameters are shown in Fig. 15. and Fig.16, and the membership function for the output is shown in Fig.17 For error (E) as input and control as output, here NL means
Negative Large, NM means Negative Medium, Z means Zero, PM means Positive Medium, and PL means Positive Large. For delta error (DE) as input, here $\mathrm{N}$ means Negative and $\mathrm{P}$ means Positive.

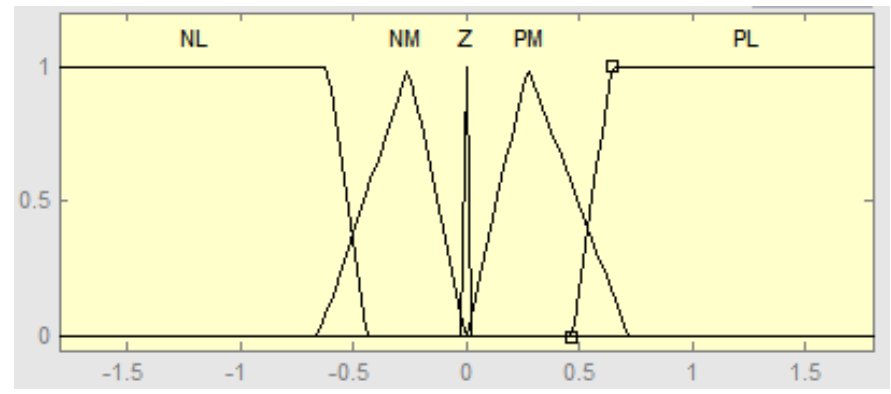

Fig.15. Membership function of error (E) as input.

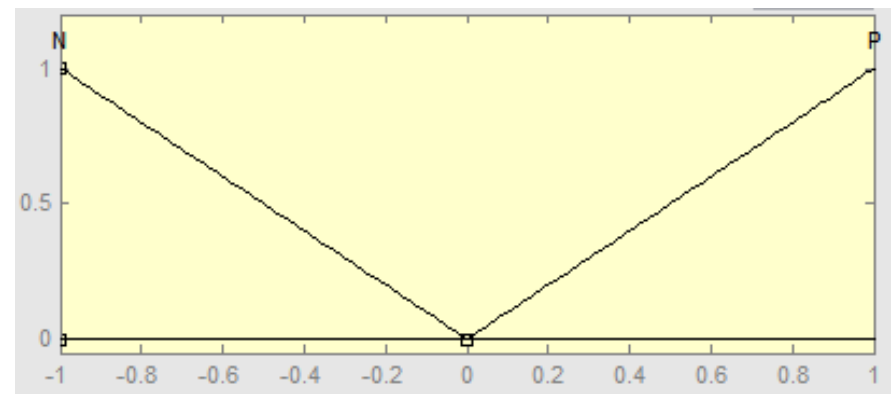

Fig.16. Membeship function of delta error (DE) as input.

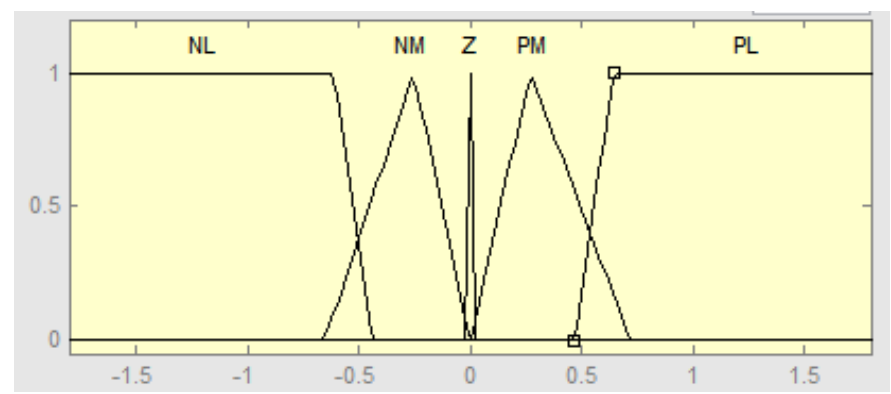

Fig.17. Membership function of CONTROL as output.

After design and construct the FIS, Fig. 18. shows the Simulink model for simulating FLC. Given $100^{\circ}$ for the final value and 0.1 second for the step time.

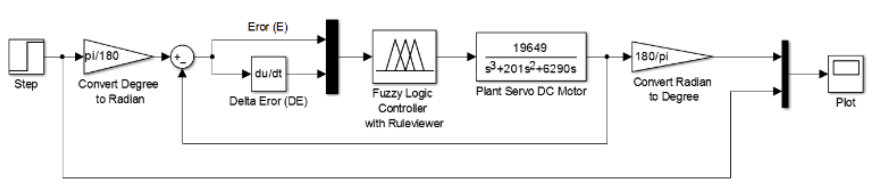

Fig.18. Simulink model for simulating FLC. 
Based on the Simulink model for simulating the FLC, Fig.19 shows the result of simulation.

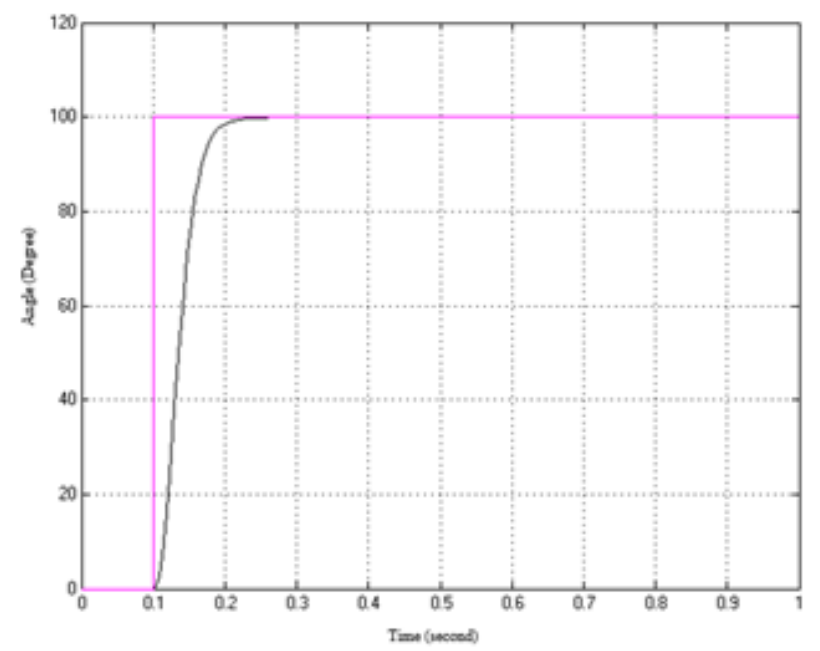

Fig. 19. Simulation result of the FLC.

Based on Fig. 19, we can get transient parameters of the system. They are a rise time, percent overshoot, settling time, and steady state error. For the system, we can get 0.07 second of the rise time (Tr), $0 \%$ of overshoot $(\mathrm{Mp}), 0.12$ second of the settling time (Ts), and $0 \%$ steady state error (Ess).

The time response parameters, including rise time $(\mathrm{Tr})$, percent overshoot (Mp), settling time (Ts) and steady state error (Ess) of the PID controller and the fuzzy logic controller (FLC) for the higher order system transfer function of DC servo motor are presented in Table II.

TABLE II. Time response parameter.

\begin{tabular}{|c|c|c|c|c|}
\hline Controller Type & $\begin{array}{c}\mathrm{Tr} \\
(\mathrm{Sec})\end{array}$ & $\begin{array}{c}\mathrm{Mp} \\
(\%)\end{array}$ & $\begin{array}{c}\mathrm{Ts} \\
(\mathrm{Sec})\end{array}$ & $\begin{array}{c}\text { (Ess) } \\
(\%)\end{array}$ \\
\hline PID Controller & 0,018 & 9,23 & 0,07 & 4 \\
\hline FLC & 0,07 & 0 & 0,12 & 0 \\
\hline
\end{tabular}

\section{CONCLUSION}

The design of DC servo motor system using both the PID controller and the FLC have been presented. Design of the PID controller has been successfully implemented but it still has an overshoot. So, the better control design has been proposed for controlling a smooth precision movement of arm robot manipulator model. Better control performance, robustness and overall stability can be expected using the FLC. The fuzzy controllers have better stability, small overshoot, and fast response. Based on the results, the time response parameters can be observed. Even though, the PID controller produces the response with lower rise time compared with the fuzzy logic controller, but it offers very high steady state error due to the oscillatory behavior in transient period. It has severe oscillations with a very high overshoot of $9.23 \%$ which causes the damage in the system performance. The proposed FLC can effectively eliminate these dangerous oscillations and provides smooth operation in transient period. Hence, it is concluded that the PID controller could not be used for the control precision movement of arm robot manipulator model because of the oscillatory. The results show that the proposed fuzzy logic based controller design can be a better choice for next implementation.

\section{REFERENCES}

[1] M. A. Rashidifar, A. A. Rashidifar, D. Ahmadi, "Modeling and Control of 5 DOF Robot Arm Using Fuzzy Logic Supervisory Control," International Journal of Robotics and Automation (IJRA), Vol. 2, No. 2, June 2013, pp. 56-68.

[2] Gaurav, Amrit Kaur, "Comparison between Conventional PID and Fuzzy Logic Controller for Liquid Flow Control: Performance Evaluation of Fuzzy Logic and PID Controller by Using MATLAB/Simulink," International Journal of Innovative Technology and Exploring Engineering (IJITEE), ISSN: 2278-3075, Volume-1, Issue-1, June 2012.

[3] C. Urrea and J Kern, "A New Model for Analog Servo Motors", Canadian Journal on Automation, Control and Intelligent Systems, Vol. 2, No. 2, March 2011.

[4] R. S . Burns, "Advances Control Engineering”, Jordan Hill, 2001.

[5] C. L. Philips and R. D. Harbor," Feedback Control Systems", PrenticeHall, 2000.

[6] K. Ogata, "Modern Control Engineering", Prentice-Hall,1970.

[7] S. N. Sivanandam, S. Sumathi and S. N. Deepa,"Introduction to Fuzzy Logic using MATLAB", Springer, 2007.

[8] S. F. Abd-Alkarim "Application of Fuzzy Logic in Servo Motor", AlKhwarizmi Engineering Journal 2007, Vol.3, No.2, pp. 8 -16.

[9] S.R.Vaishnav, Z.J.Khan, "Design and Performance of PID and Fuzzy Logic Controller with Smaller Rule Set for Higher Order System," Proceedings of the World Congress on Engineering and Computer Science, WCECS 2007, October 24-26, 2007, San Francisco, USA. 